\title{
Cell Type-Specific and Inducible PTEN Gene Silencing by a Tetracycline Transcriptional Activator-Regulated Short Hairpin RNA
}

\author{
Shan Wang ${ }^{1,2}$, Ting Wang ${ }^{1,2}$, Tao Wang ${ }^{1}$, and Lintao Jia ${ }^{1, *}$
}

\begin{abstract}
Inducible and reversible gene silencing in desired types of cells is instrumental for deciphering gene functions using cultured cells or in vivo models. However, efficient conditional gene knockdown systems remain to be established. Here, we report the generation of an inducible expression system for short hairpin RNA (shRNA) targeted to PTEN, a well-documented dual-specificity phosphatase involved in tumor suppression and ontogenesis. Upon induction by doxycycline (DOX), the reverse tetracycline transcriptional activator (rtTA) switched on the concomitant expression of GFP and a miR-30 precursor, the subsequent processing of which released the embedded PTEN-targeted shRNA. The efficacy and reversibility of PTEN knockdown by this construct was validated in normal and neoplastic cells, in which PTEN deficiency resulted in accelerated cell proliferation, suppressed apoptosis, and increased invasiveness. Transgenic mice harboring the conditional shRNAexpression cassette were obtained; GFP expression and concurrent PTEN silencing were observed upon ectopic expression of rtTA and induction with Dox. Therefore, this study provides novel tools for the precise dissection of PTEN functions and the generation of PTEN loss of function models in specific subsets of cells during carcinogenesis and ontogenesis.
\end{abstract}

\section{INTRODUCTION}

RNA interference (RNAi) has emerged as a powerful tool of gene silencing for the study of gene function and the development of novel therapeutic approaches to disease (Fellmann and Lowe, 2014; Wilson and Doudna, 2013). Compared with gene knockout at genomic loci, RNAi is a reversible method of gene silencing because switch-off of small interfering RNA (siRNA) expression restores target gene expression (Fellmann

\footnotetext{
${ }^{1}$ State Key Laboratory of Cancer Biology, Department of Biochemistry and Molecular Biology, Fourth Military Medical University, Xi'an, Shaanxi 710032 , China, ${ }^{2}$ These authors contributed equally to this work.

*Correspondence: jialth@fmmu.edu.cn
}

Received 22 May, 2015; revised 25 July, 2015; accepted 27 July, 2015; published online 19 October, 2015

Keywords: conditional gene silencing, PTEN, RNA interference, tetracycline transcriptional activator and Lowe, 2014; Kunath, 2008). Although complementary base pairing between target mRNAs and the guide strands of siRNAs provides strict specificity for gene knockdown, silencing of an individual gene in a specific type of cell or over a selected period of time, i.e., conditional gene silencing, is frequently needed (Kleinhammer et al., 2011; Wiznerowicz et al., 2006). Several siRNA or short hairpin RNA (shRNA) expression constructs are now available for cell type-specific gene knockdown (Kleinhammer et al., 2011; Wiznerowicz et al., 2006). Some of these constructs are based mainly on the Cre/loxP system, in which the Cre recombinase, driven by a specific promoter, removes a loxP-flanked spacer or transcriptional terminator to switch on shRNA expression (Fritsch et al., 2004; Hitz et al., 2007; Kasim et al., 2004; Wiznerowicz et al., 2006). The tetracycline-inducible system can also be used to exert temporal control over shRNA expression via the administration or withdrawal of tetracycline (TET) or doxycycline (DOX) (Gupta et al., 2004). Alternatively, cell specificity of DOX-induced gene knockdown can be achieved via specific promoter-dependent expression of a tetracycline transcriptional activator (tTA) or its mutant version, rtTA, which prevents or induces gene transactivation upon DOX administration, respectively (Gupta et al., 2004; Wiznerowicz et al., 2006). Of note is the generation of a Tet-regulated reproducible gene knockdown system in which Tet initiates the expression of a natural microRNA (miRNA) precursor and the subsequent miRNA splicing releases the embedded shRNA targeted to an individual gene (Dickins et al., 2007; Premsrirut et al., 2011).

Phosphatase and tensin homolog deleted on chromosome 10 (PTEN) is a potent tumor suppressor, the dysfunction of which is responsible for the occurrence of divergent human cancers (Song et al., 2012). The coordinate involvement of PTEN in embryonic development and ontogenesis has been demonstrated (Di Cristofano et al., 1998; Knobbe et al., 2008). Germline mutation of the PTEN gene in human is associated with Cowden syndrome, hyperplastic lesions in multiple organs, and neurological disorders including macrocephaly, seizure, Lhermitte-Duclos disease, and mental retardation, while conventional PTEN knockout in mice results in early embryonic death (Di Cristofano et al., 1998; Kwon et al., 2001; Li et al., 2003; Orloff and Eng, 2008; Song et al., 2012). Since the irreversible deletion of this phosphatase gene from the chromosome causes PTEN deficiency in targeted cells (e.g., stem cells) and their offspring cells, these models may have limitations in dissecting the precise role of PTEN in a specific type of 
cells during a given phase of development (Knobbe et al., 2008). In this study, we generated a conditional PTEN knockdown system, in which the PTEN-targeted shRNAs can be controlled both spatially by using a cell type-specific promoter regulating rtTA expression, and temporally via induction with DOX. The efficacy of PTEN gene silencing by this system was validated in cultured cells and a transgenic mouse model.

\section{MATERIALS AND METHODS}

\section{Plasmid and lentiviral constructs}

The pCol-TGM plasmid containing the green fluorescent protein (GFP)-miR-30 cassette flanked by tetracycline response elements (TRE) was obtained from Addgene (USA) (Premsrirut et al., 2011). The PTEN-targeted shRNA sequences were used to substitute for those of shRNA targeted to adenomatous polyposis coli (APC). Briefly, the 2 double-stranded DNA fragments, were synthesized by Shanghai Genechem Inc. (China), digested, and ligated into the Xhol/EcoRl sites of pCol-TGM. The sequences of synthesized DNAs are as follows: 5'agaaggctcgagaaggtatattgctgttgacagtgagcgaagagatcgttagcagaa acaatagtgaagccacagatgtattgtttctgctaacgatctctctgcctactgcctcgga ttcaagggg-3', and 5'-agaaggctcgagaaggtatattgctgttgaagtgagcga tgaagatgacaatcatgtttagtgaagccacagatgtaaacatgattgtcatcttcactct tactgcctcggaattcaagggg-3', which target nucleotides 18-36 and 338-356 of the coding sequence of PTEN, respectively. The resulting constructs, namely Ptn1 and Ptn2, were confirmed by DNA sequencing. A construct harboring a scrambled PTEN targeting sequence of Ptn1 was generated and used as a control. Small interfering RNAs (siRNAs), siPTEN1 and siPTEN2, were synthesized to target the aforementioned sites of PTEN. Similarly, a scrambled siRNA of siPTEN1 was included as a control. For generation of rtTA-overexpressing lentiviruses using the pLenti6.3/V5-DEST Gateway system (Invitrogen, USA), the pTet-On plasmid (ClonTech, USA) was digested with Bam$\mathrm{HI}$, blunted with T4 DNA polymerase, and digested with EcoRI. The resulting rtTA CDNA fragment was ligated into the Eco$\mathrm{RI} / E$ coRV site of the $\mathrm{pENTR}{ }^{\mathrm{TM}} 3 \mathrm{C}$ plasmid. The $\mathrm{pENTR}{ }^{\mathrm{TM}} 3 \mathrm{C}$ harboring the rtTA gene or the mCherry gene as a control was subjected to recombination with the lentiviral backbone vector and was packaged in HEK293T cells.

\section{Cell culture, treatment and transfection}

Human embryonic kidney (HEK) 293 cells, HEK293T cells, and liver carcinoma HepG2 cells were purchased from the Cell Bank of Shanghai Institute for Biological Sciences, Chinese Academy of Sciences. All cell lines were characterized by gene profiling analysis by the provider, and were used no more than 6 months after purchase. Cells were cultured in Dulbecco's modified Eagle's medium (DMEM, Gibco BRL, USA) containing $10 \%$ fetal bovine serum (FBS, Gibco BRL, USA), and maintained at $37^{\circ} \mathrm{C}$ in a humidified atmosphere containing $5 \% \mathrm{CO}_{2}$. Where indicated, cells were treated with $100 \mathrm{nM}$ insulin (Sigma, USA). Transfection of cells was performed using Turbofect transfection reagent (Thermo, USA), according to the manufacturer's instructions.

\section{Quantitative RT-PCR}

Total RNA was extracted using Trizol reagent (Invitrogen) according to the manufacturer's protocol. The reverse transcription reaction was performed with PrimeScript ${ }^{\mathrm{TM}} \mathrm{RT}$ Master Mix (TaKaRa, Japan; $1 \mu \mathrm{g}$ total RNA). The cDNAs were detected by PCR using SYBR@ Premix Ex Taq ${ }^{\mathrm{TM}}$ (TaKaRa Bio Group, Shiga, Japan). The primers used for PCR were as follows: 5'- cgtgcggataatgacaag-3' and 5'-ttgatggctcctctactg-3' for PTEN; 5'-aagaacggcatcaaggtg-3' and 5'-ctgggtgctcaggtagtgg-3' for GFP; and $5^{\prime}$-gatcattgctcctcctgagc- 3 ' and 5'-actcctgcttgctgatccac-3' for $\beta$-actin. Real-time PCR was performed in triplicate using the CFX96 Real Time PCR Detection System (Bio-Rad, USA), and the $2^{-\triangle \triangle C T}$ method was used to determine relative gene expression levels.

\section{Western blotting}

Cells were washed in phosphate buffered saline (PBS) twice before protein extraction with RIPA lysis buffer(20 mM Tris- $\mathrm{HCl}$ $\mathrm{pH} 7.4,137 \mathrm{mM} \mathrm{NaCl}, 10 \%$ glycerol, $1 \%$ Triton X-100, $0.5 \%$ sodium deoxycholate, $0.1 \%$ SDS, 2 mM EDTA pH8.0, 2 mM vanadate), and proteins were separated on a SDS/PAGE gel, transferred onto an NC membrane, and subjected to immunoblot analysis. Blotting was performed with antibodies against PTEN (Cell Signaling Technology, USA), $\beta$-actin (Cell Signaling Technology, USA), GFP (Sigma), AKT, and Ser473 phosphorylated AKT (Cell Signaling). Goat anti-rabbit and goat anti-mouse immunoglobulin horseradish peroxidase-linked $F(a b) 2$ fragments (ZB-2305, Zhong Shan Jin Qiao, China) were used as secondary antibodies.

\section{Cell proliferation assay}

Cells (3000/well) were seeded in 96-well plates in complete medium after overnight serum starvation. Cell proliferation was measured using a Cell Counting Kit-8 (CCK-8) (Boster, China). Briefly, $48 \mathrm{~h}$ after transfection the transfection medium was replaced with $100 \mu \mathrm{l}$ fresh serum-free medium with $10 \mu \mathrm{l}$ of CCK-8 solution. After incubation at $37^{\circ} \mathrm{C}$ for $1.5 \mathrm{~h}$, absorbance was measured at a wavelength of $450 \mathrm{~nm}$ in a plate reader. Cell proliferation was evaluated at $0,24,48,72$ and $96 \mathrm{~h}$ after transfection.

\section{Transwell assay of cell migration and invasion}

For invasion assay, cells were serum-starved for $6 \mathrm{~h}$ in DMEM containing $0.1 \%$ FBS. Serum-starved cells were trypsinized and resuspended in DMEM containing $0.1 \% \mathrm{BSA}$, and cells (20000/insert) were added to the upper chamber of each well coated with diluted Matrigel (Matrigel:serum-free DMEM = 1:6). After $24 \mathrm{~h}$ of incubation at $37^{\circ} \mathrm{C}$, cells on the upper membrane surface were removed by careful wiping with a cotton swab, and the filters were fixed by treatment with $95 \%$ ethanol for 20 min and stained with $0.2 \%$ crystal violet solution (Sigma) for 30 min. Cells that invaded through the pores to the lower surface of the filter were counted under a microscope. The migration assay is the same as the invasion assay except that no matrigel was used and the permeating time for cells was $14 \mathrm{~h}$.

\section{Generation of transgenic mice}

The pCol-TGM-Ptn1 construct was linearized via cleavage with $\mathrm{BamHI}$, and purified for pronuclear injection as described (Ittner and Gotz, 2007). Briefly, 300 zygotes were collected from mated C57BL6 female mice, injected with the purified DNA, and implanted into the oviduct infundibulum of the pseudopregnant foster mother. The offspring mice were screened for the integration of the transgene by genomic PCR using the following primers: 5'-cgacgtaaacggccacaagttc-3', and 5'-tcttgtagttgccg tcgtccttg-3'.

\section{Fibroblast isolation, culture, and assays}

To isolate tail-tip fibroblasts (TTFs), a $1.5 \mathrm{~cm}$ length of tail-tip from an 8-week-old mouse was washed with $70 \%$ ethanol and PBS, the superficial dermis was peeled away, and the remaining 
A

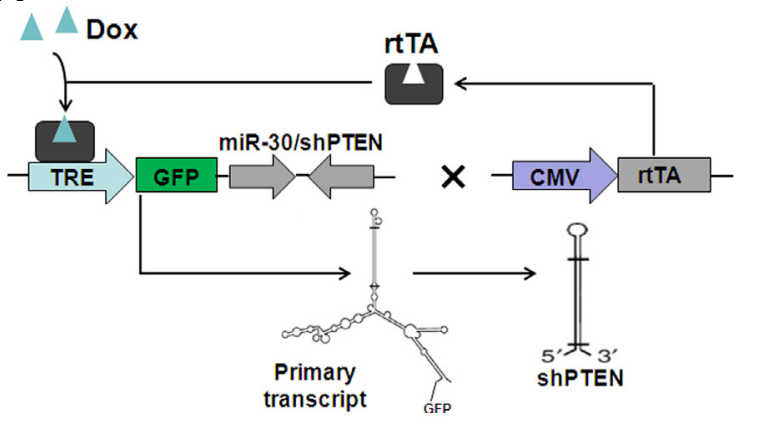

C

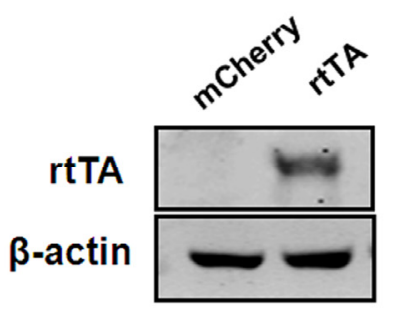

Fig. 1. Generation of the inducible expression construct for PTENtargeted shRNAs. (A) Schematic representation of the conditional knockdown system for PTEN. (B) Light (left) and fluorescent (right) microscopy showing HEK293 cells infected with control recombinant lentiviruses expressing mCherry (100x). (C) Western blot of HEK293 cells infected with the lentiviruses harboring the rtTA-expressing cassette.

B

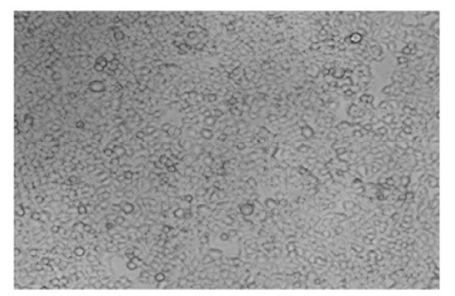

light

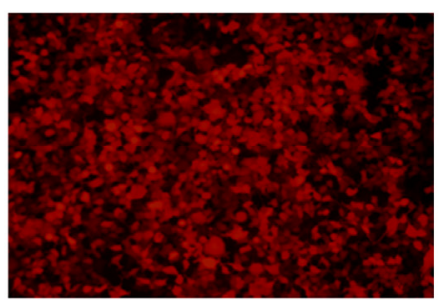

mCherry tissue was cut into 1-mm pieces using a scalpel. Five or six pieces were plated in one well of a six-well plate and cultured with $2 \mathrm{ml}$ of DMEM containing 10\% FBS and 50 units $/ \mathrm{ml}$ penicillin/streptomycin. The medium was changed every 2 to 3 days. A large number of TTFs migrated out from the explants after 5 days. Cells were then subjected to lentivirus infection, fluorescent microscopy, and Western blot assays as described above.

\section{Statistical analysis}

Statistical significance was assessed by comparing mean $( \pm$ $\mathrm{SD})$ values using Student's $t$-test for independent groups. $P \leq$ 0.05 was considered statistically significant.

\section{RESULTS}

Generation of a tetracycline-inducible siRNA expression cassette for PTEN

A previously reported conditional shRNA-expression construct based on the Tet-on system was described for PTEN targeting (Premsrirut et al., 2011). The original shRNA coding sequence for APC was replaced by those encoding shRNAs targeted to PTEN (Fig. 1A). The lentivirus vector harboring rtTA gene downstream of a constitutively active promoter was also generated, and the infection efficiency of the packaged recombinant lentiviruses were validated (Figs. $1 \mathrm{~A}$ and $1 \mathrm{~B}$ ). The lentivirusmediated overexpression of rtTA was verified in HEK293 cells (Fig. 1C). In principle, rtTA, upon activation by TET or DOX, initiates the synchronous expression of GFP and the miR-30 precursor, whose subsequent processing generates short hairpin RNAs (shRNAs) targeted to PTEN.

\section{Inducible PTEN gene silencing in normal and neoplastic} cells

To examine the efficacy of specific PTEN gene silencing in cultured cells, we stably transfected HEK293 cells and hepatic carcinoma HepG2 cells with the construct carrying shRNAs embedded in miR-30. Upon further infection with the rtTA-overexpressing lentivirus and induction with DOX, the expression of GFP was switched on and increased in a time-dependent manner in both Ptn1- and Ptn2-transfected cells (Figs. 2A and 2B). A concomitant knockdown of PTEN was observed in these cells, suggesting that the shRNAs targeted to PTEN were generated from the primary transcript containing the GFP-coding sequence and the miR-30 precursor (Fig. 2C). Similarly, upon lentivirus-mediated expression of rtTA and induction with DOX, the expression of GFP and the silencing of PTEN were achieved in HepG2 cells harboring the inducible PTEN-targeted shRNAexpressing cassette but not in cells harboring the scrambled shRNA cassette (Figs. 2D-2G). In addition, we observed a rapid restoration of $\mathrm{PTEN}$ expression in genetically modified HEK293 and HepG2 cells after withdrawal of DOX (Figs. 2B and 2E). PTEN knockdown was also observed with a delayed time course in transfectants without DOX induction, which was probably due to the presence of trace amounts of tetracycline analogues in the serum (Figs. 2B and 2E). HEK293 cells stably transfected with Ptn1, and HepG2 cells harboring Ptn2 were used thereafter for further study. The efficiency of PTEN knockdown by the DOX-inducible system was comparable with that of synthesized siRNAs (Fig. $2 \mathrm{H}$ ). Consistent with the critical role of PTEN in negatively regulating PI3K/AKT signaling, we observed significantly increased activation of AKT in cells whose PTEN was silenced by the DOX-inducible system (Fig. 2I). Taken together, the rtTA-based inducible shRNA-expression system is effective for conditional and reversible PTEN knockdown in various mammalian cells.

Inducible PTEN gene silencing promotes cell proliferation and invasion

PTEN deficiency underlies cell transformation and malignant phenotype maintenance in transformed cells (Song et al., 2012). Thus, we explored the effects of DOX-induced PTEN gene silencing on the above cells. CCK- 8 assays revealed that DOX 
A
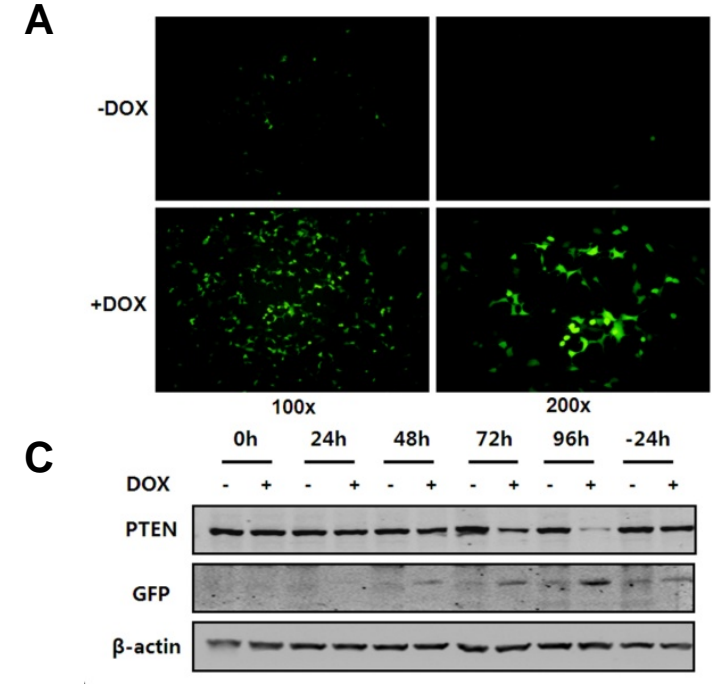

D
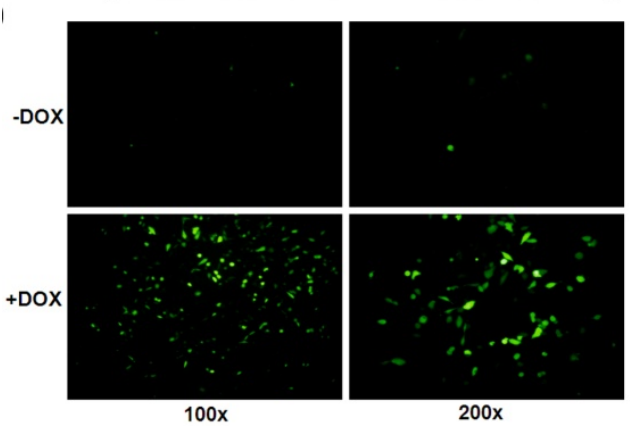

$\boldsymbol{F}$

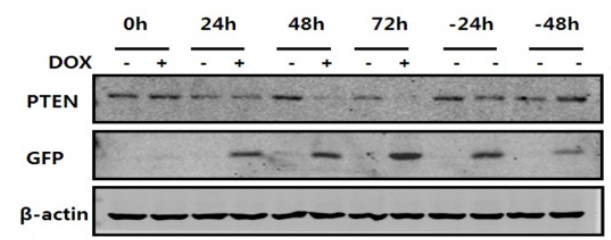

B
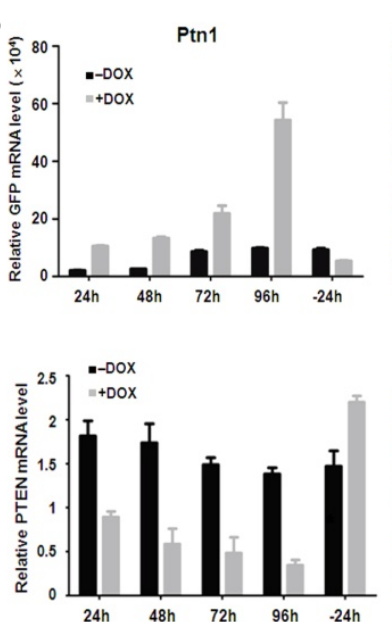

E
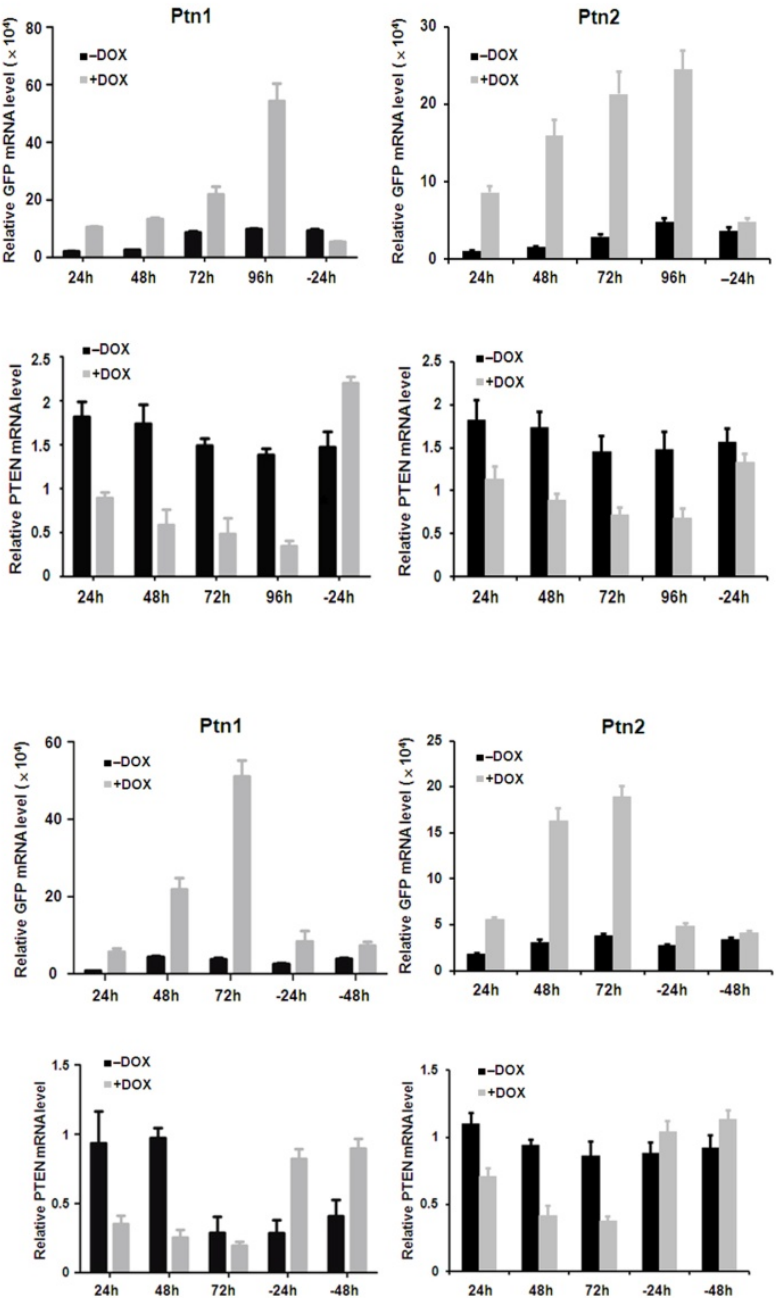

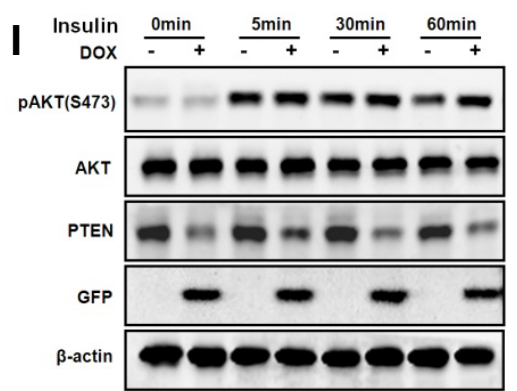

G

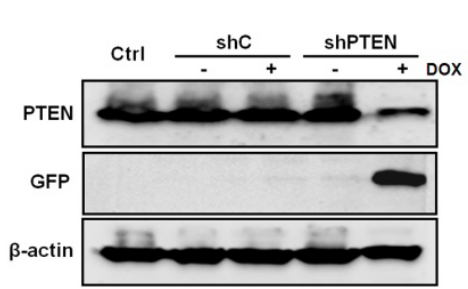

$\boldsymbol{H}$

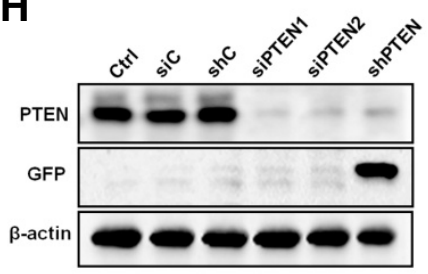

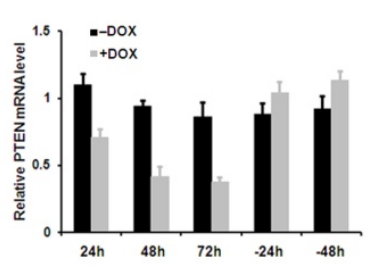

in

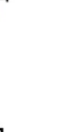


A

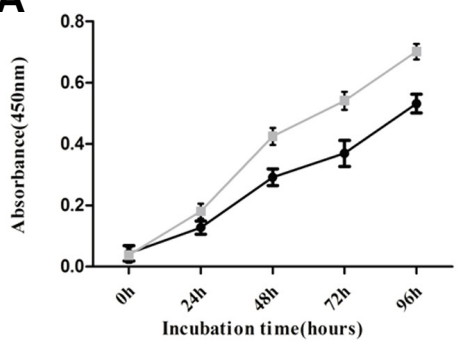

B

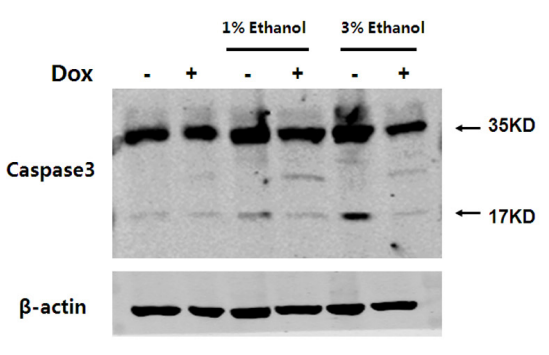

C

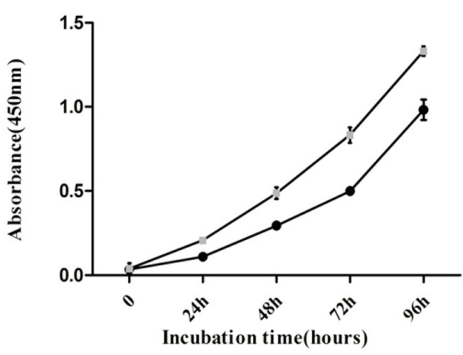

\section{D}
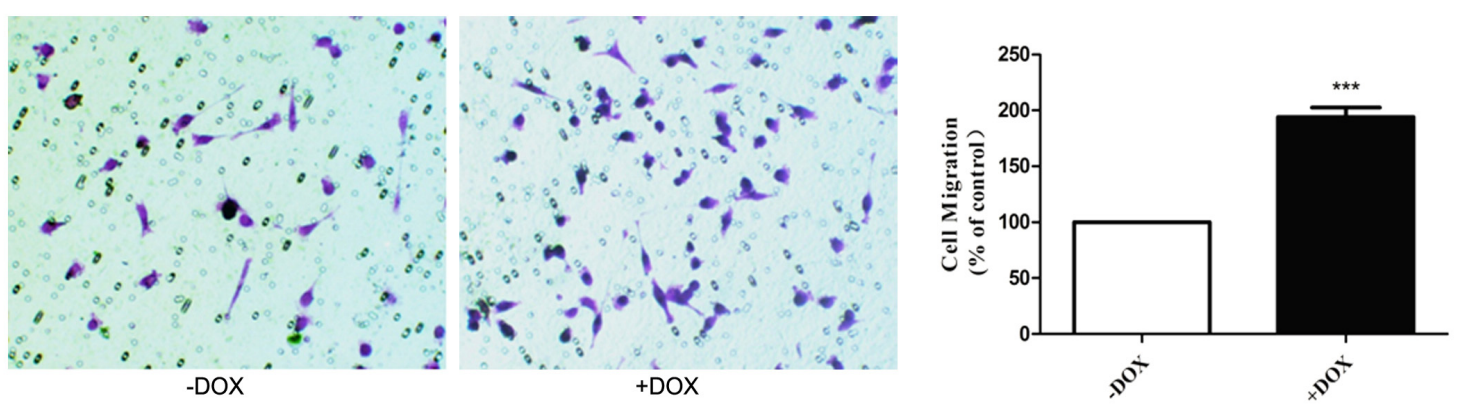

E
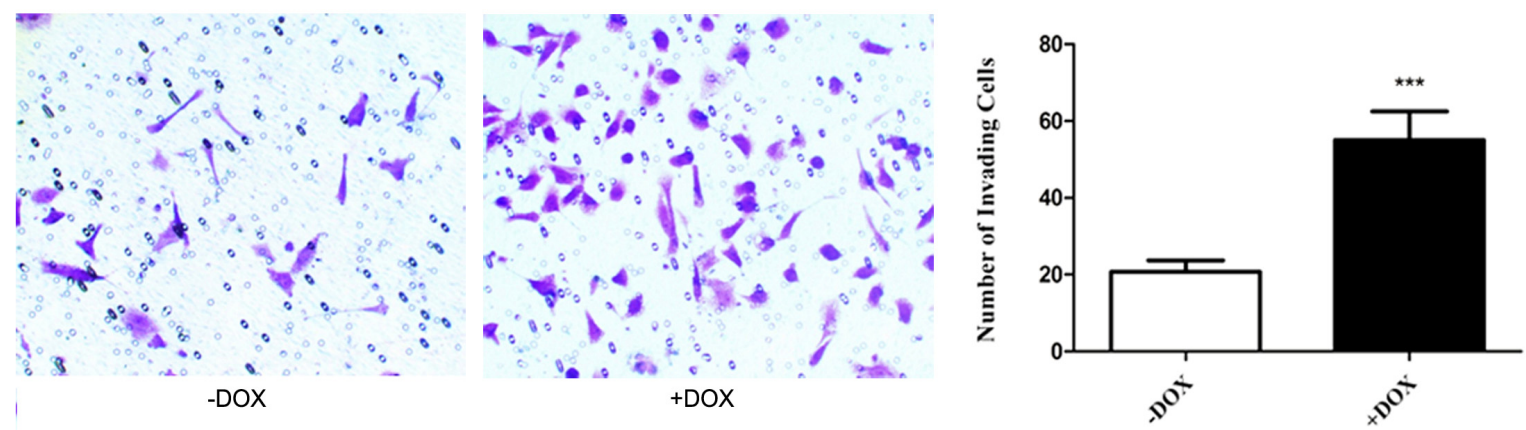

Fig. 3. Inducible PTEN knockdown promoted cell growth and invasion and suppressed apoptosis. (A, B) The stably transfected HEK293 cells described in Fig. 2A were infected with rtTA-expressing lentiviruses, followed by incubation with DOX $(1 \mu \mathrm{M})$ or the solvent as a control. CCK-8 assays (A) and Western blot analyses of cells after treatment with or without ethanol (B) were performed $48 \mathrm{~h}$ or at the indicated times of incubation with DOX. (C-E) The stably transfected HepG2 cells described in Fig. 2D were infected with rtTA-expressing lentiviruses, followed by incubation with DOX $(1 \mu \mathrm{M})$ or the solvent as a control. CCK-8 assays (C), and Transwell measurements of cell migration (D) and invasion (E) were performed $48 \mathrm{hrs}$ or at the indicated times after incubation with DOX. Data are represented as the mean $\pm S D$ of $n=3$ replicates or representative of three independent experiments. ${ }^{* \star *} P<0.001$, compared with DOX-untreated cells.

treatment caused the accelerated growth of HEK293 cells (Fig. $3 \mathrm{~A})$. DOX induction also resulted in the apparent suppression of ethanol-triggered processing and activation of caspase-3, the downstream executioner of programmed cell death (Fig. 3B). DOX-induced expression of the GFP/miR-30 transcript and the subsequent generation of PTEN-targeted shRNA in HepG2 cells resulted in increased cell proliferation (Fig. 3C), and facilitated the migration and invasion of these malignant cells (Figs. $3 \mathrm{D}$ and $3 \mathrm{E})$. These data are in agreement with the welldocumented tumor suppressor role of PTEN in various types of cells (Song et al., 2012).

\section{Specific and inducible PTEN gene silencing in transgenic} mice

The rtTA-regulated shRNA-expression cassette was then used to generate transgenic mice. After pronuclear injection of the DNA and transfer of injected zygotes into recipient foster mice, screening of offspring mice confirmed the successful incorporation of the transgene fragment in the genome (Fig. 4A). To verify the expression of GFP induced by rTTA without sacrificing the mice, we isolated the tail fibroblast cells for in vitro culture (Fig. $4 \mathrm{~B}$ ). The fibroblasts were then infected by the aforementioned recombinant lentiviruses to constitutively express rtTA. Consequently, treatment of the infected cells with DOX switched on the expression of GFP and caused downregulation of PTEN (Figs. 4C and 4D). Therefore, the transgenic mice harboring the rtTA-regulated shRNA-expression cassette would be applicable for cell type-selective PTEN silencing when hybridized with a mouse strain expressing rtTA under the control of a cell typespecific promoter. 
$\boldsymbol{A}$

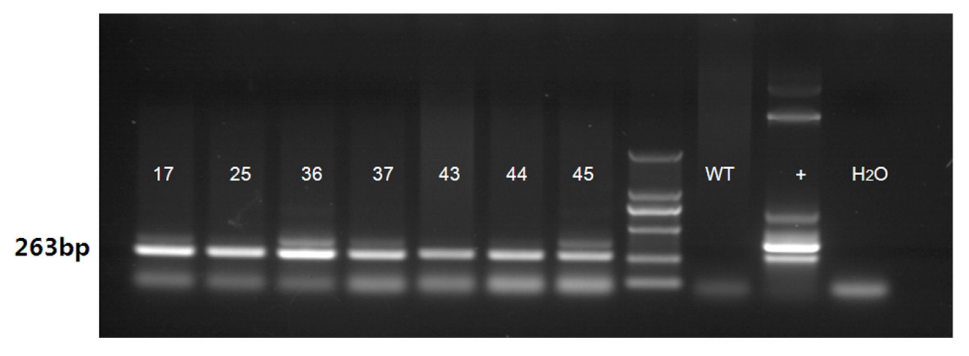

C

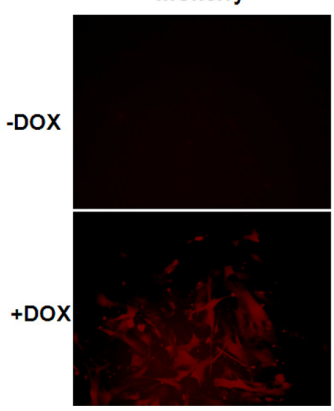

rTTA

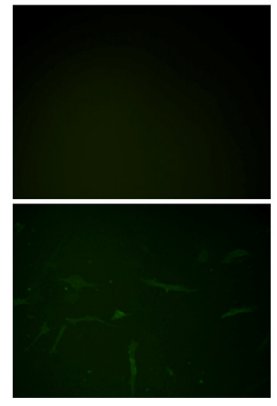

B

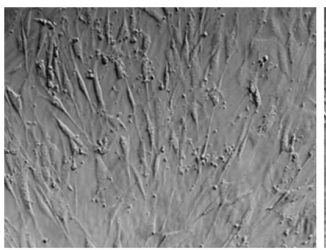

$100 x$

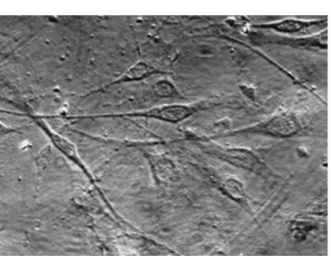

$200 X$

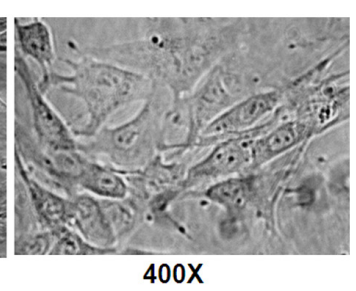

$D$

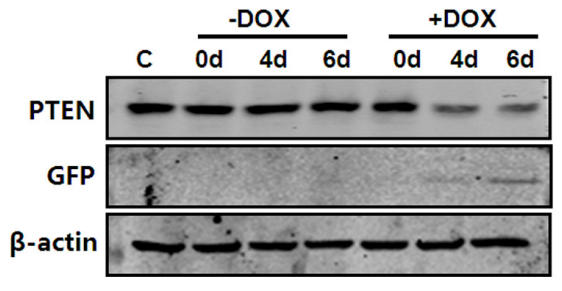

Fig. 4. Inducible PTEN silencing by the conditional shRNA-expression system in transgenic mice. The linearized pCol-TGM-Ptn1 construct was used for the generation of transgenic mice. Genomic PCR was used to screen for mice harboring the conditional shRNA-expression cassette (A). The fibroblasts were dissected from the tail of the above neonatal transgenic mice and were continuously cultured in vitro (B). Cells were infected with rtTA-expressing lentiviruses, followed by incubation with DOX $(1 \mu \mathrm{M})$ or the solvent as a control. Fluorescent microscopy (C) and Western blot analyses (D) were performed $48 \mathrm{~h}$ post-incubation with Dox or the solvent.

\section{DISCUSSION}

Past decades have witnessed the functional denotation of numerous genes using conventional or conditional gene silencing model organisms (Kleinhammer et al., 2011; Kunath, 2008). Gene loss of function is also instrumental for defining the involvement of a specific gene product in cell signaling and the regulation of the behavior of specific types of cells (Bernards, 2014; Fellmann and Lowe, 2014). Despite the development of several conditional gene knockdown systems based on Cre/loxP-mediated DNA recombination and tetracyclineresponsive transcriptional activation, the systems permitting efficient temporal and spatial control of gene silencing, especially reversible in vivo gene knockdown, remain to be developed (Kleinhammer et al., 2011; Wiznerowicz et al., 2006). Here, we achieved inducible PTEN gene knockdown by modifying a previously reported universal conditional gene silencing system (Premsrirut et al., 2011). Upon induction with DOX, rtTA initiated the concomitant expression of GFP and the miR-30 precursor, the subsequent splicing of which released the embedded shRNA targeted to PTEN. The expression of rtTA by a cell type-specific promoter in combination with this conditional shRNA-expressing cassette could be used to achieve PTEN knockdown in a given population of cells. Compared with previous conditional RNAi systems, which utilized either Cre to unblock or constitutive tTA or rtTA to transactivate shRNA expression, the current system is effective in permitting the reversible silencing of target genes in a specific subset of cells, and moreover allows detailed studies to be performed on the PTEN knockdown cells only by sorting the cells coexpressing GFP (Matthess et al., 2005; Tiscornia et al., 2004).

As a multifunctional protein and a dual-specificity phosphatase that catalyzes the dephosphorylation of phosphatidylinosi- tols and protein substrates, PTEN is involved in the regulation of a wide variety of cell events, e.g., cell cycle progression, differentiation, and apoptosis (Song et al., 2012). Nevertheless, as exemplified by the limited number of proteins that have been identified as its physiological substrates, the precise roles of PTEN in carcinogenesis, as well as in ontogenesis, remain to be determined (Hopkins et al., 2014; Song et al., 2012). For instance, PTEN plays essential roles in the development of the central nervous system (CNS), as evidenced by the enlarged neuron soma size, abnormal cell proliferation, and histoarchitectual disorganization of the brain following PTEN deletion in CNS stem cells, differentiated neurons, or specifically in granules of the cerebellum and dentate gyrus (Backman et al., 2001; Groszer et al., 2001; Kwon et al., 2001; Li et al., 2003). However, the irreversible nature of PTEN deficiency in these mice precluded using them to determine whether these abnormalities were due to a lack of PTEN in the neural stem cells or in the differentiated neurons derived from these NSCs (Groszer et al., 2001; Li et al., 2003). In this respect, the reversible PTEN knockdown system we established here could be employed to knock down PTEN selectively in NSCs, for instance, by driving rtTA expression using a NSC-specific promoter, e.g., the nestin promoter, plus administration of Dox. The conditional knockdown of PTEN could thus be stringently controlled in the littermate mice. Given the availability of mouse strains with transgenic rtTA-expressing cassettes controlled by various specific promoters, the reversible PTEN knockdown system would be applicable for the study of PTEN functions in different cell populations during the desired period of time, and would be particularly useful for determining the role PTEN plays in the intervallic developmental stage (Ko et al., 2011; Miething et al., 2014). In addition, when hybridized to mice with oncogenic mutations, these conditional PTEN deficient mice would probably develop 
malignancies in specific tissues, thus providing spontaneous tumor models with homogeneous and definitive genetic backgrounds for cancer research (Ai et al., 2014; Im et al., 2014). Taken together, the present study provides a novel approach to achieving conditional PTEN silencing, which is instrumental for precisely deciphering the function of PTEN during carcinogenesis and ontogenesis.

\section{ACKNOWLEDGMENTS}

This study was supported by the National Natural Sciences Foundation of China (No.81472631 and No. 81272646) to L.J.

\section{REFERENCES}

Ai, J., Pascal, L.E., O'Malley, K.J., Dar, J.A., Isharwal, S., Qiao, Z., Ren, B., Rigatti, L.H., Dhir, R., Xiao, W., et al. (2014). Concomitant loss of EAF2/U19 and Pten synergistically promotes prostate carcinogenesis in the mouse model. Oncogene 33, 22862294.

Backman, S.A., Stambolic, V., Suzuki, A., Haight, J., Elia, A., Pretorius, J., Tsao, M.S., Shannon, P., Bolon, B., Ivy, G.O., et al. (2001). Deletion of Pten in mouse brain causes seizures, ataxia and defects in soma size resembling Lhermitte-Duclos disease. Nat. Genet. 29, 396-403

Bernards, R. (2014). Finding effective cancer therapies through loss of function genetic screens. Curr. Opin. Genet. Dev. 24, 23-29.

Di Cristofano, A., Pesce, B., Cordon-Cardo, C., and Pandolfi, P.P. (1998). Pten is essential for embryonic development and tumour suppression. Nat. Genet. 19, 348-355.

Dickins, R.A., McJunkin, K., Hernando, E., Premsrirut, P.K., Krizhanovsky, V., Burgess, D.J., Kim, S.Y., Cordon-Cardo, C., Zender, L., Hannon, G.J., et al. (2007). Tissue-specific and reversible RNA interference in transgenic mice. Nat. Genet. 39, 914-921.

Fellmann, C., and Lowe, S.W. (2014). Stable RNA interference rules for silencing. Nat. Cell Biol. 16, 10-18.

Fritsch, L., Martinez, L.A., Sekhri, R., Naguibneva, I., Gerard, M., Vandromme, M., Schaeffer, L., and Harel-Bellan, A. (2004). Conditional gene knock-down by CRE-dependent short interfering RNAs. EMBO Rep. 5, 178-182.

Groszer, M., Erickson, R., Scripture-Adams, D.D., Lesche, R., Trumpp, A., Zack, J.A., Kornblum, H.I., Liu, X., and Wu, H. (2001). Negative regulation of neural stem/progenitor cell proliferation by the Pten tumor suppressor gene in vivo. Science 294 , 2186-2189.

Gupta, S., Schoer, R.A., Egan, J.E., Hannon, G.J., and Mittal, V. (2004). Inducible, reversible, and stable RNA interference in mammalian cells. Proc. Natl. Acad. Sci. USA 101, 1927-1932.

Hitz, C., Wurst, W., and Kuhn, R. (2007). Conditional brain-specific knockdown of MAPK using Cre/loxP regulated RNA interference. Nucleic Acids Res. 35, e90.

Hopkins, B.D., Hodakoski, C., Barrows, D., Mense, S.M., and Parsons, R.E. (2014). PTEN function: the long and the short of it.
Trends Biochem. Sci. 39, 183-190.

Im, E., Jung, J., Pothoulakis, C., and Rhee, S.H. (2014). Disruption of Pten speeds onset and increases severity of spontaneous colitis in II10(-/-) mice. Gastroenterology 147, 667-679 e610.

Ittner, L.M., and Gotz, J. (2007). Pronuclear injection for the production of transgenic mice. Nat. Protoc. 2, 1206-1215.

Kasim, V., Miyagishi, M., and Taira, K. (2004). Control of siRNA expression using the Cre-loxP recombination system. Nucleic Acids Res. 32, e66.

Kleinhammer, A., Deussing, J., Wurst, W., and Kuhn, R. (2011). Conditional RNAi in mice. Methods 53, 142-150.

Knobbe, C.B., Lapin, V., Suzuki, A., and Mak, T.W. (2008). The roles of PTEN in development, physiology and tumorigenesis in mouse models: a tissue-by-tissue survey. Oncogene 27, 5398-5415.

Ko, J.K., Choi, K.H., Zhao, X., Komazaki, S., Pan, Z., Weisleder, N., and $\mathrm{Ma}$, J. (2011). A versatile single-plasmid system for tissuespecific and inducible control of gene expression in transgenic mice. FASEB J. 25, 2638-2649.

Kunath, T. (2008). Transgenic RNA interference to investigate gene function in the mouse. Methods Mol. Biol. 461, 165-186.

Kwon, C.H., Zhu, X., Zhang, J., Knoop, L.L., Tharp, R., Smeyne, R.J., Eberhart, C.G., Burger, P.C., and Baker, S.J. (2001). Pten regulates neuronal soma size: a mouse model of LhermitteDuclos disease. Nat. Genet. 29, 404-411.

Li, L., Liu, F., and Ross, A.H. (2003). PTEN regulation of neural development and CNS stem cells. J. Cell. Biochem. 88, 24-28.

Matthess, Y., Kappel, S., Spankuch, B., Zimmer, B., Kaufmann, M. and Strebhardt, K. (2005). Conditional inhibition of cancer cell proliferation by tetracycline-responsive, $\mathrm{H} 1$ promoter-driven silencing of PLK1. Oncogene 24, 2973-2980.

Miething, C., Scuoppo, C., Bosbach, B., Appelmann, I., Nakitandwe J., Ma, J., Wu, G., Lintault, L., Auer, M., Premsrirut, P.K., et al. (2014). PTEN action in leukaemia dictated by the tissue microenvironment. Nature 510, 402-406.

Orloff, M.S., and Eng, C. (2008). Genetic and phenotypic heterogeneity in the PTEN hamartoma tumour syndrome. Oncogene 27 5387-5397.

Premsrirut, P.K., Dow, L.E., Kim, S.Y., Camiolo, M., Malone, C.D., Miething, C., Scuoppo, C., Zuber, J., Dickins, R.A., Kogan, S.C., et al. (2011). A rapid and scalable system for studying gene function in mice using conditional RNA interference. Cell 145 145-158.

Song, M.S., Salmena, L., and Pandolfi, P.P. (2012). The functions and regulation of the PTEN tumour suppressor. Nature reviews. Mol. Cell Biol. 13, 283-296.

Tiscornia, G., Tergaonkar, V., Galimi, F., and Verma, I.M. (2004). CRE recombinase-inducible RNA interference mediated by lentiviral vectors. Proc. Natl. Acad. Sci. USA 101, 7347-7351.

Wilson, R.C., and Doudna, J.A. (2013). Molecular mechanisms of RNA interference. Annu. Rev. Biophys. 42, 217-239.

Wiznerowicz, M., Szulc, J., and Trono, D. (2006). Tuning silence: conditional systems for RNA interference. Nat. Methods 3, 682688. 